\title{
Kinetic-free safe operation of fine chemical runaway reactions:
}

\section{a general criterion.}

\author{
Francesco Maestri, Renato Rota* \\ Politecnico di Milano \\ Dip. di Chimica, Materiali e Ingegneria Chimica "G. Natta" \\ Piazza Leonardo da Vinci, 32 - 20133 Milano - Italy \\ fax: +39 0223993180; e-mail: renato.rota@polimi.it
}

\begin{abstract}
$\underline{\text { Abstract }}$
In this work a simple and general method has been developed that, on the basis of an easy to measure quantity referred to as the $\Psi$ number, allows for on-going detecting the displacement of the SBR operating regime from the safe target conditions, without any information about the reaction kinetics.

Such a feature is of particular importance since, when dealing with fine-chemical and pharma reaction processes, the estimation of the kinetic parameters of the reactions involved is often not practicable, because of the huge variety of productions encountered and the simultaneous occurrence of mass transfer phenomena in heterogeneous systems.

Moreover, the method in question has been proved to be useful for an early detection of low reactivity or not ignition regimes of the SBR, in order to prevent dangerous accumulation phenomena resulting in a thermal loss of control of the main reaction and in a further triggering of pressure generating decomposition events.

The criterion has been finally validated analyzing the available data of an industrial SBR in which a water emulsion polymerization of acrylic monomers is performed.
\end{abstract}

\section{Keywords:}

Kinetics; Safety; Semibatch reactor (SBR); Pseudo-steady-state regime; $\Psi$ number; Scale-up; Early warning; Polymerization.

\footnotetext{
* to whom correspondence should be addressed
} 


\section{Introduction}

In the fine chemical and pharma industry a number of non-continuous reaction processes undergo safety problems due to potentially runaway reactions that can cause dangerous reactor overpressures $^{1}$. In these cases the reaction should be better performed in a semi-batch-reactor (SBR) operated under the so called target conditions, that is, at a minimum coreactant accumulation and at a high reactor cooling efficiency ${ }^{2-4}$.

In order to minimize the coreactant accumulation in the system, a dosing time much higher than the characteristic time of the reaction must be adopted, the latter being directly related to the process macrokinetics. However, due to the huge number of reactions involved, the kinetic parameters are often unknown and in any case not straightforward to be characterized, also because of the simultaneous occurrence of mass transfer phenomena in multi-phase systems ${ }^{2-4,5,6}$.

In the process safety literature of the last thirty years a number of safety criteria have been developed, allowing for selecting safe operating conditions of exothermic SBRs, without solving the mathematical model of the reactor ${ }^{2-4,7,8,9,10-12}$. However, it must be noticed that the safe operating regime of an exothermic SBR, even if it can be identified through a comparison between coreactant dosing time and reaction characteristic time, corresponds, once reached, to conditions in which the conversion rate is fully determined by the coreactant supply and is therefore independent on the reaction macrokinetics.

In this work a general method has been developed that, through an easy to measure quantity referred to as the $\Psi$ number, allows for on-going detecting the displacement of the SBR operating conditions from a pseudo-steady-state regime with respect to both the coreactant accumulation and the reaction temperature, corresponding to the aforementioned target conditions. Under such a regime, the $\Psi$ number approaches univocally a constant value equal to 100 , which is independent on the SBR operating parameters. The approach of the SBR operating regime to such conditions can therefore be directly controlled during the reactor operation through simply adjusting the coreactant supply rate, so as to reach $\Psi$ number values close to 100 yet in the first fraction of the dosing period. 
The on-going measurement of the $\Psi$ number can be easily performed, through a simple combination of flowrate and temperature measurements, which are normally already available for any industrial SBR. Moreover, the criterion in question can be regarded as an early warning detection system to prevent dangerous accumulation phenomena in exothermic SBRs ${ }^{12}$, due to unexpected reactivity drops or sudden reaction inhibitions, which would be otherwise difficult to recognize during the normal reactor operation.

The proposed criterion has been successfully validated analyzing the available process data of a SBR in which a water emulsion polymerization of acrylic monomers is performed.

\section{Mathematical model}

Developing a general safety criterion for isoperibolic SBRs in which potentially runaway reactions are performed requires a preliminary discussion about the target conditions for the safe reactor operation, presented elsewhere in the literature ${ }^{2-4,9}$.

It is assumed that a single reaction of general power law kinetics is performed, either in a homogeneous or in a heterogeneous SBR, where the coreactant A is added at a constant flowrate to a previously loaded amount of reactant $\mathrm{B}$. The mass balance equation for the i-th species is:

$\frac{d n_{i}}{d t}=F_{i, d o s} \pm v_{i} r^{e f f} V_{r}$

where $\mathrm{F}_{\mathrm{i} \text {,dos }}$ differs from zero for A only and the production term at the right hand side is positive or negative if the i-th species is a reaction product or a reactant, respectively. The effective reaction rate, $\mathrm{r}^{\text {eff }}$, depends on the microkinetic rate expression and, for heterogeneous systems, also on the phase in which the reaction takes place as well as on the controlling step among the chemical reaction and the interphase mass transfer. At the beginning of the supply period the number of moles in the reactor, $\mathrm{n}_{\mathrm{i}, 0}$, differs from zero for species B only.

The energy balance equation for the SBR is:

$\left(n \tilde{C}_{P}\right) \frac{d T}{d t}=F_{d o s} \tilde{C}_{P, d o s}\left(T_{d o s}-T\right)+V_{r} r^{e f f}(-\Delta \widetilde{H})-U A\left(T-\bar{T}_{\text {cool }}\right)$ 
stating that the reaction temperature time profile is the result of three enthalpy contributions, related to the dosing stream, the chemical reaction and the heat removal by the coolant. At the beginning of the supply period the SBR is at a temperature $T_{0}$, which is assumed to be equal to the coolant temperature, $\mathrm{T}_{\text {cool }}$.

The numerical integration of equations (1) and (2) with the related initial conditions provides the time profiles of the SBR composition and temperature.

The target conditions for the safe reactor operation (also referred to in the mentioned literature as QFS, that is, Quick onset, Fair conversion, and Smooth temperature profile conditions) state that:

- the coreactant dosing time is much higher than the macrokinetic characteristic time of the reaction. Under such conditions the dosed coreactant is consumed by the chemical reaction at a much lower time scale than that at which it is supplied. As a consequence the coreactant accumulation in the system is effectively limited and quickly approaches a pseudo-steadystate behavior, according to which the mass balance equation for species A simplifies as follows:

$\frac{d n_{A}}{d t}=F_{A, d o s}-v_{A} r^{e f f} V_{r} \approx 0$

- the characteristic time of the heat evolution by the chemical reaction is much higher than that related to the heat removal by the external coolant and the feed stream. Under such conditions the reaction temperature increase due to the exothermic reaction occurring is counteracted at a much lower time scale by the heat removal contribution. As a consequence the reactor temperature approaches a pseudo-steady-state behavior as well, according to which the SBR energy balance simplifies as follows:

$$
\left(n \tilde{C}_{P}\right) \frac{d T}{d t}=F_{d o s} \tilde{C}_{P, \text { dos }}\left(T_{\text {dos }}-T\right)+V_{r} r^{e f f}(-\Delta \widetilde{H})-U A\left(T-\bar{T}_{\text {cool }}\right) \approx 0
$$

- analogously, since the coolant residence time in the jacket or coil is usually negligible (mainly because of the relatively low coolant hold-up in the jacket or coil itself), the average coolant temperature increase due to the heat transfer from the reactor is counteracted at a 
much lower time scale by the increase of the outlet coolant temperature. Therefore, also the coolant temperature approaches a pseudo-steady-state behavior:

$$
\begin{aligned}
& \tau_{\text {cool }} \frac{d \bar{T}_{\text {cool }}}{d t}=\frac{U A}{\dot{M}_{\text {cool }} \hat{C}_{P, \text { cool }}}\left(T-\bar{T}_{\text {cool }}\right)-\left(T_{\text {cool,ouT }}-T_{\text {cool,IN }}\right) \approx 0 \\
& \tau_{\text {cool }}=m_{\text {cool }} / \dot{M}_{\text {cool }} \text { being the coolant residence time in the jacket or coil. }
\end{aligned}
$$

Combining equation (4) with the energy balance equation for the coolant (5), the following relationship can be derived:

$F_{\text {dos }} \tilde{C}_{P, \text { dos }}\left(T-T_{\text {dos }}\right)+\dot{M}_{\text {cool }} \hat{C}_{P, \text { cool }}\left(T_{\text {cool,out }}-T_{\text {cool, IN }}\right) \cong V_{r} r^{\text {eff }}(-\Delta \widetilde{H})$

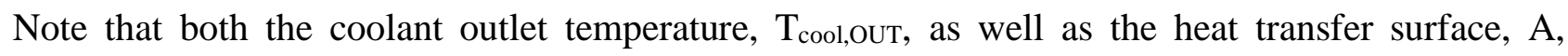
increase with time. Substituting equation (3) in equation (6), the following expression can be obtained, which holds true under target conditions:

$F_{\text {dos }} \tilde{C}_{P, \text { dos }}\left(T-T_{\text {dos }}\right)+\dot{M}_{\text {cool }} \hat{C}_{P, \text { cool }}\left(T_{\text {cool }, \text { out }}-T_{\text {cool }, I N}\right) \cong \frac{F_{A, \text { dos }}}{v_{A}}(-\Delta \widetilde{H})$

Under such target conditions the overall heat removal rate from the system (through both the external coolant and the dosing stream) defined as:

$\dot{Q}_{\text {cool }}=F_{\text {dos }} \tilde{C}_{P, \text { dos }}\left(T-T_{\text {dos }}\right)+\dot{M}_{\text {cool }} \hat{C}_{P, \text { cool }}\left(T_{\text {cool }, \text { oUT }}-T_{\text {cool }, I N}\right)$

is therefore equal to:

$\dot{Q}_{c o o l, t a}=\frac{F_{A, d o s}}{v_{A}}(-\Delta \widetilde{H})$

Defining a new parameter, called the $\Psi$ number, as the ratio of the overall heat removal rate (8) to its target value (9):

$\Psi=\frac{F_{\text {dos }} \tilde{C}_{P, \text { dos }}\left(T-T_{d o s}\right)+\dot{M}_{\text {cool }} \hat{C}_{P, \text { cool }}\left(T_{\text {cool }, O U T}-T_{\text {cool }, I N}\right)}{\frac{F_{A, \text { dos }}}{v_{A}}(-\Delta \widetilde{H})} \times 100$

it is evident that under target conditions $\Psi_{\mathrm{ta}}=100$. From a physical point of view the $\Psi$ number can therefore be regarded as an index of the approach, during the dosing period, of the SBR operation to a pseudo-steady-state regime with respect to both the coreactant accumulation and the reaction temperature, to which the aforementioned target conditions correspond. In particular, reaching 
relatively high $\Psi$ numbers in the first fraction of the supply period allows for early limiting the coreactant accumulation in the system under high cooling efficiency operating conditions.

It must be finally noticed that the on-going measurement of the $\Psi$ number can be easily performed knowing just the reaction enthalpy and combining two flowrate measurements (that is, the feed stream and the coolant flowrate measurement) and two temperature difference measurements (that is, the coolant temperature increase through the jacket or coil and the difference between reactor and feed stream temperatures), which are typically already available for any industrial SBR.

\section{Different SBR behaviors}

The $\Psi$ number introduced in the previous section allows for distinguishing the different SBR operating regimes: an SBR in which an exothermic reaction is performed has to be regarded as both a reaction system and a heat transfer equipment, so that its safe operation must take into account for both the phenomena involved, that is, the chemical reaction and the reactor cooling. The $\Psi$ number accounts indeed for both the chemical reaction ignition and the efficiency of the heat removal by the external coolant and the dosing stream: in particular, a relatively high $\Psi$ number in the first fraction of the supply period corresponds to an SBR operating regime in which the coreactant accumulation is minimized and the reactor cooling efficiency is maximized.

Adopting SBR operating conditions characterized by both a relatively low coreactant accumulation and a relatively high heat removal efficiency, the reaction temperature approaches all along the supply period the corresponding target profile $^{2-4}$. Moreover, the target temperature profile (and hence the reaction temperature) undergoes a relatively small variation during the supply period itself, because of the high heat removal efficiency. As a consequence, even if the SBR is operated under isoperibolic conditions at a constant feed and coolant flowrate, the chemical reaction occurs during the supply period under nearly isothermal conditions that would otherwise be more complicated to keep through manipulating the feed and/or the coolant flowrate. 
Such an SBR operation, which can be automatically reached by early adopting relatively high $\Psi$ numbers, is desirable for most of the reaction processes that for chemical reasons (for instance, related to the final product quality) must be performed within a narrow range around a given temperature.

In Figure 1 the $\Psi$ number and temperature time profiles for a well ignited-high cooling efficiency SBR (calculated through numerical solution of equations (1) and (2) with proper initial conditions) have been plotted: the characteristics and operating parameters refer to an industrial organic nitration through mixed acids, as summarized in Table 1. Under such operating regime the characteristic time of the conversion rate (and hence of its enthalpy effects) is close to the dosing time and much higher than the characteristic time of the heat removal. As a consequence, the exothermic reaction contribution is instantaneously balanced by the reactor cooling, and the $\Psi$ number quickly reaches values close to 100 that are then kept for most of the supply period.

As the feed is stopped the reaction enthalpy contribution disappears with a minimum delay, due to the relatively low coreactant accumulation in the system. Therefore, the reaction temperature drops to the external coolant temperature and the heat removal contribution as well as the $\Psi$ number go asymptotically to zero.

Analogously, the reaction temperature quickly reaches its target profile and stays close to it up to the end of the supply period, with a minimum variation across an average value.

When searching for safe operating conditions of an exothermic SBR, the process variables to be manipulated are the dosing time and the initial reaction temperature. However, the reaction temperature is normally defined by the chemical recipe, since it has a significant influence on the purity of the final product, according to its specifications. Therefore, the only process variable which is really at the engineer's disposal is the coreactant dosing time.

In Figure 2 the $\Psi$ number time profiles for the same process summarized in Table 1 at different coreactant dosing times are represented. It can be noticed that as a higher dosing time is adopted, the SBR reaches higher $\Psi$ number values at a lower fraction of the whole dosing period, the target 
limit being univocally equal to 100, independently on the SBR operating parameters. Moreover, at the lower dosing times the $\Psi$ number time profile undergoes a more evident discontinuity at the end of the supply period, that is, when the enthalpy contribution of the dosing stream disappears: such a behavior is related to the higher entity of this contribution at the higher feed rates.

Analogously, in Figure 3 the temperature time profiles for different dosing times are represented. It can be observed that the higher the dosing time is, the earlier the target temperature profile is reached. However, as a relevant difference with respect to the $\Psi$ number behavior, the target temperature profile depends on the SBR operating parameters and in particular on the coreactant dosing time. Such a result is logical since, when a higher dosing time is adopted, the reaction heat evolution is spread over a wider period and hence lower peak temperatures are reached for a given heat transfer efficiency of the reaction equipment.

Instead, adopting the $\Psi$ number as a controlled variable, as a higher dosing time is adopted, the $\Psi$ number tends univocally to reach values close to 100 for a higher fraction of the dosing period. Therefore, adopting the $\Psi$ number as a controlled variable, it is straightforward to adjust the feed rate so as to reach relatively high $\Psi$ numbers in the first fraction of the dosing period, which corresponds to the aforementioned target regime for the safe SBR operation.

\section{Practical use of the $\Psi$ number}

As previously discussed, the $\Psi$ number computation during the supply period is based on heat transfer measurements that are performed through flowrate and temperature difference measurements. Typically a flowrate measurement is subject to a constant relative error, $\varepsilon_{\mathrm{F}}$, of the order of $0.2 \%$, if it is performed through conventional mass flowmeters operating within their proper range ${ }^{13}$; a temperature measurement is instead typically subject to a constant absolute error, $\mathrm{T}_{\varepsilon}$, depending on the employed device and on the temperature range. When measuring a temperature difference, it is therefore evident that the relative error, $\varepsilon_{\Delta \mathrm{T}}$, increases as the temperature difference to be measured decreases, according to the following expression: 
$\varepsilon_{\Delta T}=\frac{2 T_{\varepsilon}}{\Delta T} \times 100$

$2 \mathrm{~T}_{\varepsilon}$ being the maximum absolute error to be expected for a temperature difference measurement.

Therefore, at the industrial scale, it is recommended to limit the coolant flowrate so as to keep during the dosing period a temperature increase of this fluid through the reactor jacket or coil not lower than $2^{\circ} \mathrm{C}$. In this way, employing resistance temperature detectors (RTDs) for temperature measurements, typically having accuracies $\mathrm{T}_{\varepsilon} \sim 0.1^{\circ} \mathrm{C}{ }^{13}$, the relative error on the measured temperature difference would be lower than $10 \%$. As a consequence, the accuracy on the $\Psi$ number measurement can be estimated through the following expression:

$\frac{\left(1-\varepsilon_{F}\right)\left(1-\varepsilon_{\Delta T}\right)}{1+\varepsilon_{F}} \Psi \leq \Psi \leq \frac{\left(1+\varepsilon_{F}\right)\left(1+\varepsilon_{\Delta T}\right)}{1-\varepsilon_{F}} \Psi$

With the industrial accuracies on the flowrate and temperature difference measurements stated above, a roughly $10 \%$ maximum error on the $\Psi$ number measurement can be expected as well.

In order to easily improve such an accuracy, a redundancy can be adopted on each temperature measurement (that is, on the coolant inlet and outlet as well as on the reactor temperature and dosing stream temperature). In this way a statistical error reduction on each temperature measurement and therefore on the final temperature difference measurement can be achieved.

Moreover, it is advisable to perform before each batch a system calibration consisting in setting the temperature measured by all the instruments at the same value, that is, at the initial system temperature $\mathrm{T}_{0}$ : in this way also the influence of any heat loss from the system can be offset. For what concerns the dosing stream enthalpy contribution in the $\Psi$ number expression, if the coreactant is dosed at the same temperature as the initial reaction one, the aforementioned temperature calibration procedure can be easily extended to the dosing vessel. If instead the dosing stream temperature is far below the initial reaction one, the percentage error on the difference between reaction and dosing stream temperatures will be automatically low all along the process, because of the relatively high difference between the two involved temperatures. Adopting the 
aforementioned plant solutions, an accuracy in the $\Psi$ number measurement better than $5 \%$ can be easily achieved.

In order to analyze the influence of the $\Psi$ number on the SBR operating conditions, it is useful to introduce the following normalized sensitivity coefficient ${ }^{14}$ :

$S=\frac{\langle\Psi\rangle}{\phi} \frac{\partial \phi}{\partial\langle\Psi\rangle} \approx \frac{\langle\Psi\rangle}{\phi} \frac{\Delta \phi}{\Delta\langle\Psi\rangle}$

where $\phi=\mathrm{T}_{\max } / \mathrm{T}_{0}$ is the ratio of the peak to the initial reaction temperature, and $\langle\Psi\rangle=$ $\frac{1}{t_{d o s}} \int_{0}^{t_{d o s}} \Psi(t) d t$ is the average $\Psi$ number during the supply period.

In Figure 4 the functional dependence of $S$ on $\langle\Psi>$ has been represented for the simulated SBR. It can be noticed that the normalized sensitivity coefficient, S, increases with $<\Psi>$, mainly in the $<\Psi>$ range of industrial interest, that is, above 70. In particular, taking into account that the orders of magnitude of $\left\langle\Psi>\right.$ and $\phi$ are $10^{2}$ and $10^{\circ}$ respectively, it can be estimated that for the analyzed nitration process at the higher $\left\langle\Psi>\right.$ values, $\Delta \phi / \Delta<\Psi>$ is close to $4 \cdot 10^{-3}$ : since $\Delta<\Psi>$ is of the order of $10^{1}$ (which is close to its maximum range of error for conventionally instrumented SBRs), it can be concluded that $\Delta \phi$ is close to $4 \cdot 10^{-2}$, to which a peak temperature variation equal to about $13^{\circ} \mathrm{C}$ corresponds. This means that a small $<\Psi>$ variation is related to a peak temperature variation of the order of $10^{\circ} \mathrm{C}$, which on the contrary is relatively high. It can therefore be concluded that the $\Psi$ number is a suitable process variable to select safe operating conditions for the SBR and to monitor any displacement of the SBR regime with respect to them.

\section{Scale-up through the $\Psi$ number}

The proposed criterion can be applied to the selection of safe operating conditions of exothermic SBRs at the laboratory or pilot plant scale and to the further scale-up of such operating conditions to the industrial plant. It must be stressed that the developed procedure is based on an experimental identification of the target conditions for the safe reactor operation through the $\Psi$ number, that is, 
through an easy to measure process variable reaching under such conditions a well-defined target value, independently on the SBR operating parameters.

Since under this operating regime the reaction macrokinetics has a negligible influence on the conversion rate (which is at the limit fully determined by the coreactant supply rate) this criterion does not require a kinetic characterization of the system. Such a feature is of particular importance when dealing with a wide number of reaction processes, such as those encountered in the fine chemical and pharma industry. At both the scales (that is, at the laboratory as well as at the industrial scale) only the equipment geometry and heat transfer characteristics must be known, the latter being easy to estimate through simple calibration tests. Moreover, the physical properties of the involved products and mixtures as well as the reaction heat must be estimated: however, this requires a much lower effort than that required for characterizing the system macrokinetics.

Performing several runs at the laboratory or pilot plant scale with different feed rates and recording the $\Psi$ number time profiles during the supply period, it is straightforward to identify the maximum feed rate allowing for reaching high $\Psi$ number values in the first fraction of the dosing period, the latter two quantities (that is, the threshold $\Psi$ number value and the time at which it must be reached) being arbitrarily fixed.

It is evident that the earlier the selected measure time and the higher the desired $\Psi$ number are, the higher the dosing time is. For what concerns the selection of a $\Psi$ threshold value, it must be noticed that as the reaction ignition as well as the reactor heat removal efficiency drop, the system typically undergoes a well-detectable $\Psi$ number decrease: it is therefore not convenient from a productivity point of view to adopt too high $\Psi$ number threshold values (and hence correspondingly high dosing times) for the selection of safe operating conditions of the SBR.

For what concerns the selection of the time at which one requires the $\Psi$ number value to be larger than the selected threshold, a relatively early detection is recommended. In fact, when facing for the sake of example an unexpected reaction inhibition, the earlier the $\Psi$ number is detected and 
compared with its threshold value, the lower the coreactant accumulation in the system is: therefore, the adopted corrective actions (typically consisting in a feed interruption) will be more effective in order to keep the process safe. Moreover, the earlier the detection time is, the higher the dependence of the measured $\Psi$ number on the adopted dosing time is.

Such a behavior is represented in Figure 5, where the $\Psi$ numbers measured at different fractions of the dosing period are plotted vs. the adopted dosing time for the case study summarized in Table 1. It can be noticed that as the measure time is shifted towards the middle of the coreactant supply, the detected $\Psi$ number reaches a nearly asymptotic behavior with respect to the dosing time, which makes more difficult to recognize the $\Psi$ number increase with the selected dosing time, according to the accuracy of the available instruments for the heat removal measurements. On the contrary, when an early measure time is selected, a significant dependence of the detected $\Psi$ number on the adopted dosing time can be achieved.

As a rule of thumb, on the basis of the expected industrial accuracy of the $\Psi$ number measurements previously discussed, a $\Psi$ number lower limit between 80 and 100 can be reasonably adopted; moreover, the $\Psi$ number measurement can be performed e.g. at the $20 \%$ of the dosing time, provided that the incidental accumulation of the $20 \%$ of the dosed coreactant cannot in any case imply peak temperatures higher than the Maximum Allowable Temperature (MAT) for the current reaction process: otherwise, an earlier detection time can be adopted.

Assuming that the same macrokinetic regime (slow or fast reaction) prevails both at the lab or pilot plant scale and at the industrial scale, the scale-up of the operating conditions identified for the laboratory reactor can be safely performed adopting at both the scales the same Westerterp number, according to the criterion presented elsewhere in the literature ${ }^{9,15}$ :

$W t_{L A B}=\left.\frac{(U A)_{0} t_{d o s}}{\varepsilon\left(\widetilde{\rho} \tilde{C}_{P} V_{r}\right)_{0}}\right|_{L A B}=W t_{I N D}=\left.\frac{(U A)_{0} t_{d o s}}{\varepsilon\left(\widetilde{\rho} \tilde{C}_{P} V_{r}\right)_{0}}\right|_{I N D}$

Since the initial heat transfer surface per unit volume, (UA) $)_{0} / \mathrm{V}_{\mathrm{r} 0}$, is typically lower for the industrial reactor (varying roughly with the reciprocal of the equipment diameter for jacketed vessels) the 
scaled-up dosing time will be correspondingly higher. Moreover, the laboratory scale dosing time is indeed already much higher than the macrokinetic characteristic time of the reaction, since it arises from experimentally identified operating conditions to which a sufficiently high measured $\Psi$ number corresponds.

It must be noticed that the macrokinetic characteristic time of the reaction, $\tau_{\mathrm{r}, \mathrm{MC}}$, is nearly equal at the two scales: for homogeneous and heterogeneous systems operating in the slow reaction regime, this statement holds true without any approximation, since the aforementioned characteristic time (which is equal to the microkinetic one) depends just on concentrations and temperatures that do not change at the two scales; for heterogeneous systems operating in the fast reaction regime the same statement is realistic, assuming that at both the scales an equally efficient mixing is guaranteed.

Therefore it can be concluded that the scale-up to the industrial SBR of the operating conditions experimentally identified at the laboratory scale is safe, being:

$t_{d o s, I N D}>t_{d o s, L A B} \gg \tau_{r, M C}$

and provided that keeping constant the Wt number accounts for the different cooling efficiency.

\section{SBR monitoring through the $\Psi$ number}

Once the safe operating conditions have been experimentally identified for the laboratory or pilot equipment and further scaled-up to the industrial SBR, the value of the dosing time at the industrial scale can be fine-tuned by monitoring the $\Psi$ number value during the dosing period and enforcing the aforementioned constraints (for instance, $\Psi \geq 80$ at $t=0.2 t_{\text {dos }}$ ). Moreover, the on-going measurement of the $\Psi$ number during the coreactant supply period allows for monitoring the safe reactor operation during each successive batch of a productive campaign.

In fact, a drop of the system reactivity (due, for the heterogeneous nitration previously discussed, for instance to a stirring system failure, if not otherwise detected) as well as of the heat removal efficiency leads to a well-detectable decrease of the $\Psi$ number, even during the coreactant supply (see for the sake of example Figure 6, where the effects on the $\Psi$ number profile of a sudden 
reactivity drop occurring in the middle of the supply period has been represented). This allows for a prompt corrective action (typically consisting in a feed interruption), aimed to counteract the coreactant accumulation in the system.

In the process safety literature of the last twenty years, a number of criteria have been developed to early detect the triggering of runaway phenomena in non-continuous exothermic reactors ${ }^{12}$. Such criteria, through space reconstruction techniques, allow for identifying an incipient thermal explosion of the system, after the causes of the reaction loss of control have occurred.

The on-going measurement of the $\Psi$ number during the coreactant supply period can instead be regarded as an a-priori detection criterion of the aforementioned causes, that is, of anomalous coreactant accumulation phenomena as well as of drops of the heat removal efficiency. However, once safe SBR operating conditions have been correctly selected and scaled-up, it is unlikely that from one batch to another of a productive campaign a drop of the heat transfer efficiency occurs for reasons related to the chemical process: drops of the reactor heat removal efficiency are instead typically due to plant failures (for instance, a coolant circulation breakdown) that, however, can be easily detected in a well instrumented plant.

On the contrary, process disturbances that are likely to originate dangerous coreactant accumulations can occur randomly and are much more difficult to be promptly detected and counteracted through suitable corrective actions: examples of such process anomalies are catalyst loading errors due to human factors or inhibitions of the chemical reaction due to incidental leakages (for instance, air or water) into the reactor, which can occur at the beginning as well as during the dosing period. These would lead to an unexpected drop of the $\Psi$ number during the supply period.

Therefore, through on-going measuring the $\Psi$ number it is possible to early detect dangerous accumulation phenomena in exothermic SBRs that, arising from not evident plant or process anomalies, would be otherwise difficult to be promptly identified. 
Once the process anomaly has been detected, a plant corrective action can be triggered, typically consisting in a feed interruption. In any case it must be stressed that the adoption of the described early warning detection system as well as of any other plant instrumentation aimed to early detect runaway phenomena cannot at all replace the installation on the involved plant sections of passive protection systems (that is, of pressure safety valves and/or rupture disks), properly designed to relieve the system overpressure in the worst case scenario.

\section{Case study: water emulsion polymerization of acrylic monomers}

The described criterion has been tested through the analysis of some data of an industrial SBR in which a potentially runaway reaction (that is, the water emulsion polymerization of acrylic monomers) occurs. The reaction involves methacrylic acid (MA) and ethyl acrylate (EA) as main monomers (co-polymerized with minor amounts of heavier species) and must be performed dosing the monomer water emulsion at a sufficiently low rate, so as to minimize the monomers accumulation in the reactor and to keep the reaction temperature within its proper range (that is, $85 \div 90^{\circ} \mathrm{C}$ ). The reaction is fairly exothermic and, if not properly controlled, can cause reactor overpressures due to the reaction mass boiling ${ }^{16}$.

In particular, on the basis of the polymerization heats of the two monomers (equal to $768 \mathrm{~kJ} / \mathrm{kg}$ for MA and to $655 \mathrm{~kJ} / \mathrm{kg}$ for EA) and of the organic monomer phase composition (corresponding to the 44.9\% weight for both MA and EA, the remaining part being related to the above mentioned heavy co-monomers) an average monomer phase polymerization heat equal to $639 \mathrm{~kJ} / \mathrm{kg}$ can be estimated.

On the basis of available literature data ${ }^{17}$, an average heat capacity of the dosed water emulsion equal to $2.867 \mathrm{~kJ} /\left(\mathrm{kg}^{\circ} \mathrm{C}\right)$ can be calculated; moreover, a heat capacity and density of the coolant equal to $3.89 \mathrm{~kJ} /\left(\mathrm{kg}^{\circ} \mathrm{C}\right)$ and $1010 \mathrm{~kg} / \mathrm{m}^{3}$ can be estimated.

Recording the relevant plant data for the application of the criterion in question (that is, the coolant flowrate and inlet/outlet temperatures, the feed flowrate and temperature, and the reactor temperature) it is straightforward to derive the $\Psi$ number time profile, represented in Figure 7 . It 
can be observed that, after a quick reactor onset, the $\Psi$ number reaches values close to 90 , which are kept for the whole duration of the supply period, with a standard deviation lower than 5\%.

On the basis of the $\Psi$ number values, the reaction is expected to be performed at a low unreacted monomers accumulation and at a sufficiently high cooling efficiency. This is confirmed by the reaction temperature varying within a relatively narrow range across its initial value. Such evidences are in agreement with the industrial experience on the reaction in question, according to which the adopted operating conditions lead to a nearly quantitative monomer conversion at the end of the 6 hours supply period: in fact, half an hour after the monomer feed stop, the unconverted EA concentration in the reaction mass is typically lower than 1000ppm.

Therefore, the selected operating regime for the SBR could have been experimentally identified through simple $\Psi$ number measurements, performed in a laboratory or pilot equipment and finally scaled-up to the industrial plant according to the described criterion, without any information about the system macrokinetics.

Moreover, when dealing with acrylic polymerizations, unexpected reaction inhibitions can occur because of e.g. auxiliary chemicals loading errors or air leakages into the reactor: such phenomena, if not promptly detected, can cause even dangerous unreacted monomers build-ups in the system, which can then trigger thermal explosion events and reactor overpressures. Also in this case the ongoing monitoring of the $\Psi$ number during the supply period is useful for easily detecting the reaction inhibition and for promptly triggering a corrective action aimed to stop the monomers feed, hence keeping the process safe.

\section{8. $\underline{\text { Conclusions }}$}

When dealing with potentially runaway reactions performed in a SBR, a safe reactor operation can be achieved adopting a sufficiently low supply rate, so that the dosed coreactant is consumed by the chemical reaction at a much lower time scale than that at which it is fed, and the heat removal efficiency from the system is relatively high. Under such conditions the SBR operation approaches a pseudo-steady-state regime with respect to both the corectant accumulation and the reaction 
temperature. However, selecting a suitable dosing time through its comparison with the macrokinetic characteristic time of the reaction, requires the measurement of the reaction kinetic parameters, which is often complicated when dealing with fine chemical and pharma reaction processes, because of the huge number of productions involved.

In this work a general criterion has been developed, through which the aforementioned safe conditions can be experimentally identified without any kinetic information about the involved chemical reactions.

The method is based on an easy to measure quantity, referred to as the $\Psi$ number, which is an index of the approximation of the SBR operating conditions to the aforementioned pseudo-steady-state regime. Such a quantity can be computed from a simple combination of process variables that are typically known for any well-instrumented plant.

Moreover, this criterion can be adopted to early detect unexpected reaction inhibitions during the supply period, which are likely to cause dangerous coreactant accumulations if a prompt corrective action (mainly, a feed stop) is not triggered.

The criterion has been finally validated using some plant data of an industrial SBR in which a potentially runaway polymerization is performed. 


\section{Nomenclature}

The units indicated for the listed quantities are valid for the equations reported in the paper but could change in the plotted results according to the reported legends.

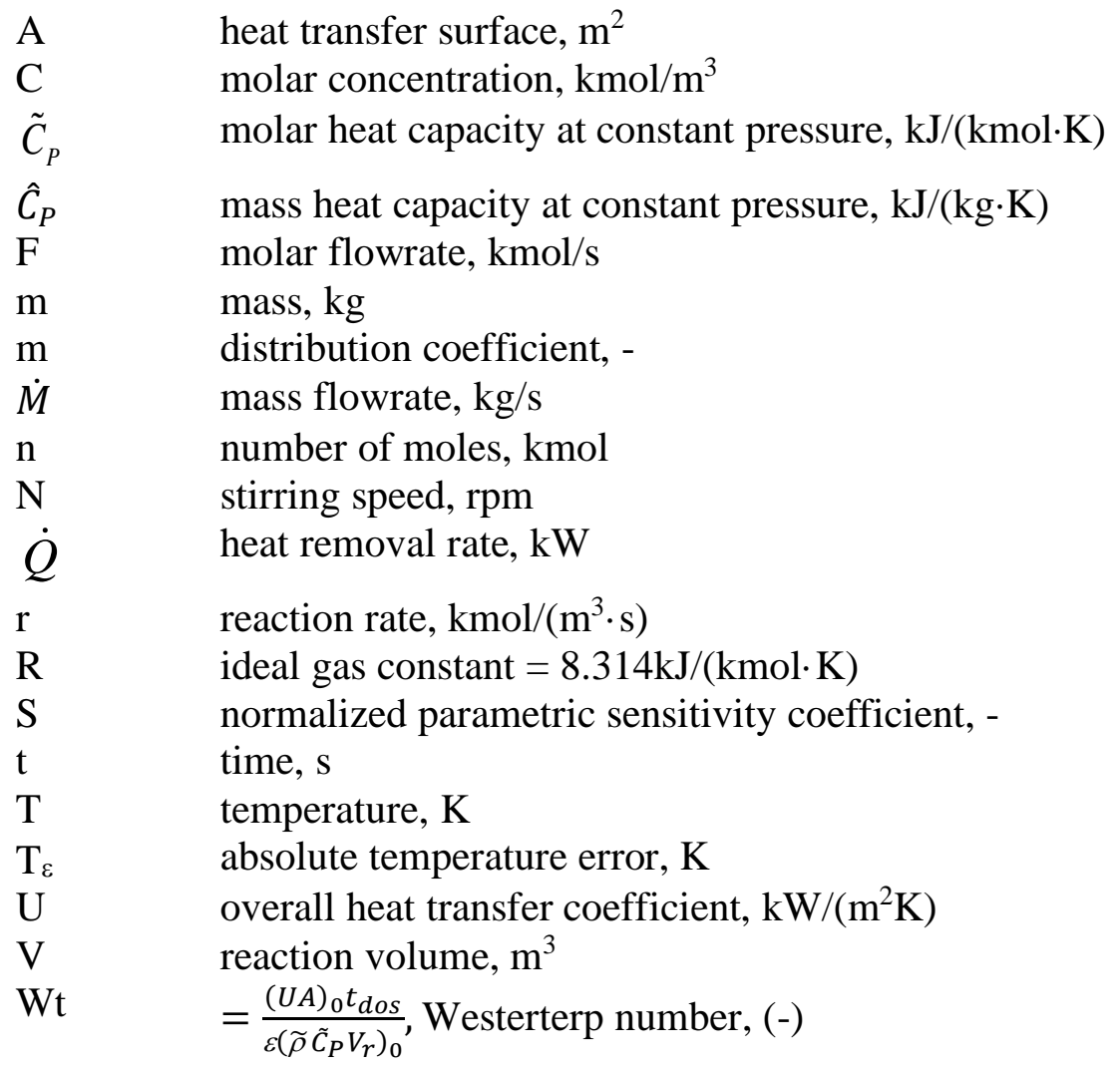

Subscripts and superscripts

\begin{tabular}{|c|c|}
\hline $\begin{array}{l}\mathrm{A} \text { and } \mathrm{B} \\
\text { cool }\end{array}$ & $\begin{array}{l}\text { components } \mathrm{A} \text { and } \mathrm{B} \\
\text { coolant }\end{array}$ \\
\hline c & continuous phase \\
\hline d & dispersed phase \\
\hline dos & dosing stream or dosing time \\
\hline eff & effective \\
\hline $\mathrm{F}$ & in $\varepsilon_{\mathrm{F}}$ \\
\hline$\Delta \mathrm{T}$ & in $\varepsilon_{\Delta \mathrm{T}}$ \\
\hline $\mathrm{i}$ & i-th component \\
\hline IN & inlet \\
\hline IND & industrial \\
\hline LAB & laboratory \\
\hline $\max$ & maximum \\
\hline meas & measure \\
\hline $\mathrm{MC}$ & macrokinetic \\
\hline OUT & outlet \\
\hline $\mathrm{r}$ & reaction \\
\hline $\mathrm{R}$ & reactor \\
\hline ta & target \\
\hline
\end{tabular}


0 start of the semibatch period

$\varepsilon \quad$ in $\mathrm{T}_{\varepsilon}$

Greek symbols

$\begin{array}{ll}\Delta \tilde{H} & \text { reaction enthalpy, } \mathrm{kJ} / \mathrm{kmol} \\ \Delta \mathrm{T} & \text { temperature difference, } \mathrm{K} \\ \varepsilon & =\mathrm{V}_{\text {dos }} / \mathrm{V}_{\mathrm{r} 0} \text {, relative volume increase, - } \\ \varepsilon & \text { relative error, - } \\ \vartheta & =\mathrm{t} / \mathrm{t}_{\mathrm{dos}}, \text { dimensionless time, - } \\ \nu & \text { stoichiometric coefficient, - } \\ \tilde{\rho} & \text { molar density, } \mathrm{kmol} / \mathrm{m}^{3} \\ \hat{\rho} & \text { mass density, } \mathrm{kg} / \mathrm{m}^{3} \\ \tau & \text { characteristic time or residence time, } \mathrm{s} \\ \phi & =\mathrm{T}_{\max } / \mathrm{T}_{0}, \text { peak to initial reaction temperature ratio, - } \\ \Psi & \Psi \text { number, see Equation (10) }\end{array}$




\section{$\underline{\text { References }}$}

(1) Steinbach, J. Safety Assessment for Chemical Processes. Wiley-VCH, Weinheim, 1999.

(2) Steensma, M.; Westerterp, K.R. Thermally safe operation of a cooled semi-batch reactor. Slow liquid-liquid reactions. Chem. Eng. Sci. 1988, 43, Nr.8, 2125-2132.

(3) Steensma, M.; Westerterp, K.R. Thermally safe operation of a semibatch reactor for liquidliquid reactions. Slow reactions. Ind. Eng. Chem. Res. 1990, 29, 1259-1270.

(4) Steensma, M.; Westerterp, K.R. Thermally safe operation of a semibatch reactor for liquidliquid reactions. Fast reactions. Chem. Eng. Technol. 1991, 14, 367-375.

(5) Maestri, F.; Rota, R. Thermally safe operation of liquid-liquid semibatch reactors. Part I: single kinetically controlled reactions with arbitrary reaction order. Chem. Eng. Sci. 2005, 60, 3309-3322. (6) Maestri, F.; Rota, R. Thermally safe operation of liquid-liquid semibatch reactors. Part II: single diffusion controlled reactions with arbitrary reaction order. Chem. Eng. Sci. 2005, 60, 5590-5602. (7) Hugo, P.; Steibach, J. Praxisorientierte Darstellung der thermischen Scherheitsgrenzen für den indirekt gekühlten Semibatch-Reaktor. Chem. Ing. Tech. 1985, 57, Nr. 9, 780-782.

(8) Hugo, P.; Steinbach, J. A comparison of the limits of safe operation of a SBR and a CSTR.

Chem. Eng. Sci. 1986, 41, 1081-1087.

(9) Maestri, F.; Copelli, S.; Rota, R.; Lunghi, A.; Gigante, L.; Cardillo, P. A simple procedure for optimal scale-up of fine chemical processes. Part I: practical tools. Ind. Eng. Chem. Res. 2009, 48 (3), 1307-1315.

(10) Copelli, S.; Derudi, M.; Maestri, F.; Rota, R. Safe operating conditions for semibatch processes involving consecutive reactions with autocatalytic behavior. Chem. Eng. Sci. 2010, 65, 5464-5470. (11) Copelli, S.; Derudi, M.; Cattaneo, C.S.; Nano, G.; Raboni, M.; Torretta, V.; Rota, R. Synthesis of 4-chloro 3-nitro benzotrifluoride: industrial thermal runaway due to cooling system failure. Process Safety and environmental protection 2014, 92, 659-668.

(12) Copelli, S.; Torretta, V.; Pasturenzi, C.; Derudi, M.; Cattaneo, C.S.; Rota, R. On the divergence criterion for runaway detection: application to complex controlled systems. Journal of 
loss prevention in the process industries 2014, 28, 92-100.

(13) Perry, R.H.; Green, D.W. Chemical engineers’ handbook. Mc Graw Hill, 2008.

(14) Varma, A.; Morbidelli, M., Wu, H. Parametric Sensitivity in Chemical Systems. Cambridge University Press, 1999.

(15) Pohorecki, R.; Molga, E. Letter to the Editor: the Westerterp number (Wt). Chem. Eng. Res. and Des. 2010, 88, 385-387.

(16) Etchell, J.; Wilday, J. Workbook for chemical reactor relief system sizing. Health and Safety Executive, 1998.

(17) Yaws, C.L. Chemical Properties Handbook. Mc Graw Hill, 1998. 


\section{Captions to the figures}

Figure 1. Well ignited - high cooling efficiency SBR. Parameter values as in Table 1. (A) $\Psi$ number vs. time; (B) Reactor temperature vs. time.

Figure 2. $\Psi$ number vs. time at increasing dosing times for an SBR. Parameter values as in Table 1 (except the dosing time).

Figure 3. Reactor temperature vs. time at increasing dosing time for an SBR. Parameter values as in Table 1 (except the dosing time).

Figure 4. Normalized sensitivity coefficient of the peak to initial temperature ratio, $\phi$, with respect to the average $\Psi$ number during the supply period. Parameter values as in Table 1 (apart from the dosing time).

Figure 5. $\Psi$ number computed at different fractions of the dosing period vs. dosing time. Parameter values as in Table 1 (except the dosing time).

Figure 6. $\Psi$ number vs. time with sudden reaction inhibition occurring in the middle of the dosing period. Parameter values as in Table 1.

Figure 7. Measured $\Psi$ number vs. time for water emulsion acrylic polymerization in an industrial SBR. 


\begin{tabular}{|c|c|c|}
\hline $\begin{array}{l}\text { Quantity } \\
\hat{\rho}_{c}\end{array}$ & $\begin{array}{l}\text { Units } \\
\left(\mathrm{kg} / \mathrm{m}^{3}\right)\end{array}$ & $\begin{array}{l}\text { Value } \\
1787\end{array}$ \\
\hline$\hat{C}_{P, c}$ & $\left(\mathrm{~kJ} / \mathrm{kg}^{\circ} \mathrm{C}\right)$ & 1.477 \\
\hline$\hat{\rho}_{d}$ & $\left(\mathrm{~kg} / \mathrm{m}^{3}\right)$ & 1353 \\
\hline$\hat{C}_{P, d}$ & $\left(\mathrm{~kJ} / \mathrm{kg}^{\circ} \mathrm{C}\right)$ & 0.869 \\
\hline$\Delta \widetilde{H}_{r}$ & $(\mathrm{~kJ} / \mathrm{kmol})$ & -123000 \\
\hline$C_{B 0, c}$ & $\left(\mathrm{kmol} / \mathrm{m}^{3}\right)$ & 2.764 \\
\hline $\mathrm{V}_{\mathrm{c}}\left(=\mathrm{V}_{\mathrm{r} 0}\right)$ & $\mathrm{m}^{3}$ & 4.688 \\
\hline $\mathrm{V}_{\text {dos }}$ & $\mathrm{m}^{3}$ & 1.641 \\
\hline$V_{R}$ & $\mathrm{~m}^{3}$ & 9 \\
\hline$A_{0}$ & $\mathrm{~m}^{2}$ & 45 \\
\hline$(\mathrm{UA})_{0}$ & $\mathrm{~kW} / \mathrm{K}$ & 12 \\
\hline $\mathrm{N}$ & rpm & 200 \\
\hline $\mathrm{T}_{0}$ & ${ }^{\circ} \mathrm{C}$ & 60 \\
\hline $\mathrm{T}_{\text {cool }}$ & ${ }^{\circ} \mathrm{C}$ & 60 \\
\hline $\mathrm{T}_{\text {dos }}$ & ${ }^{\circ} \mathrm{C}$ & 60 \\
\hline $\mathrm{t}_{\text {dos }}$ & $\mathrm{h}$ & 3 \\
\hline
\end{tabular}

Table 1. Characteristics and operating parameters of an SBR in which an organic species, A, is added to nitric acid, $\mathrm{B}$, mixed with sulfuric acid. The reaction, occurring in the continuous phase and in the slow-reaction regime, can be described through the rate expression $r=$ $k_{0} e^{-\frac{E}{R T}} m_{A} C_{A, d} C_{B, c}$ with $\mathrm{k}_{0}=3.228 \cdot 10^{12} \mathrm{~m}^{3} /(\mathrm{kmol} \cdot \mathrm{s}), \mathrm{m}_{\mathrm{A}}=10^{-2}, \mathrm{E}=87260 \mathrm{~kJ} / \mathrm{kmol}$. 


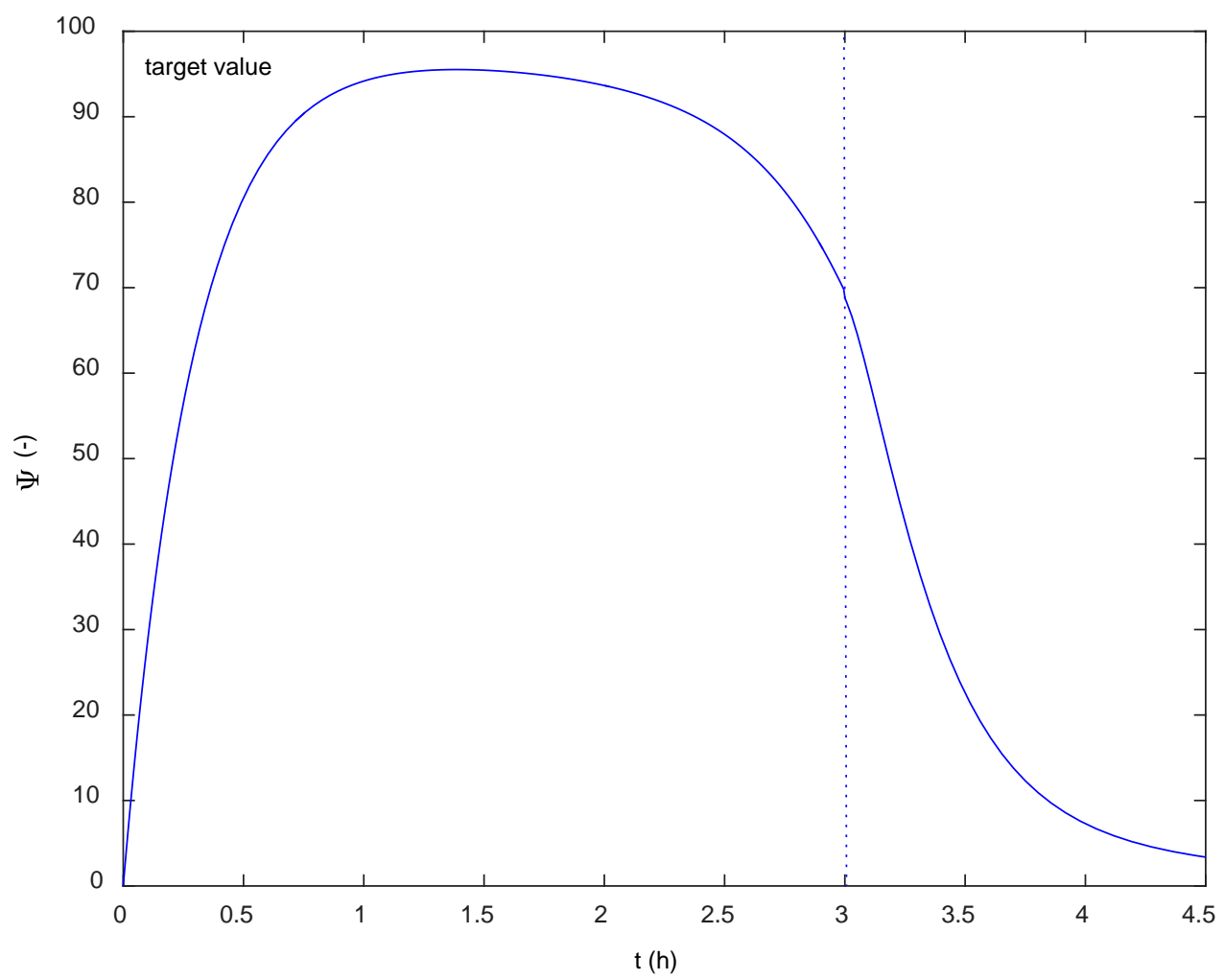

Fig. 1A

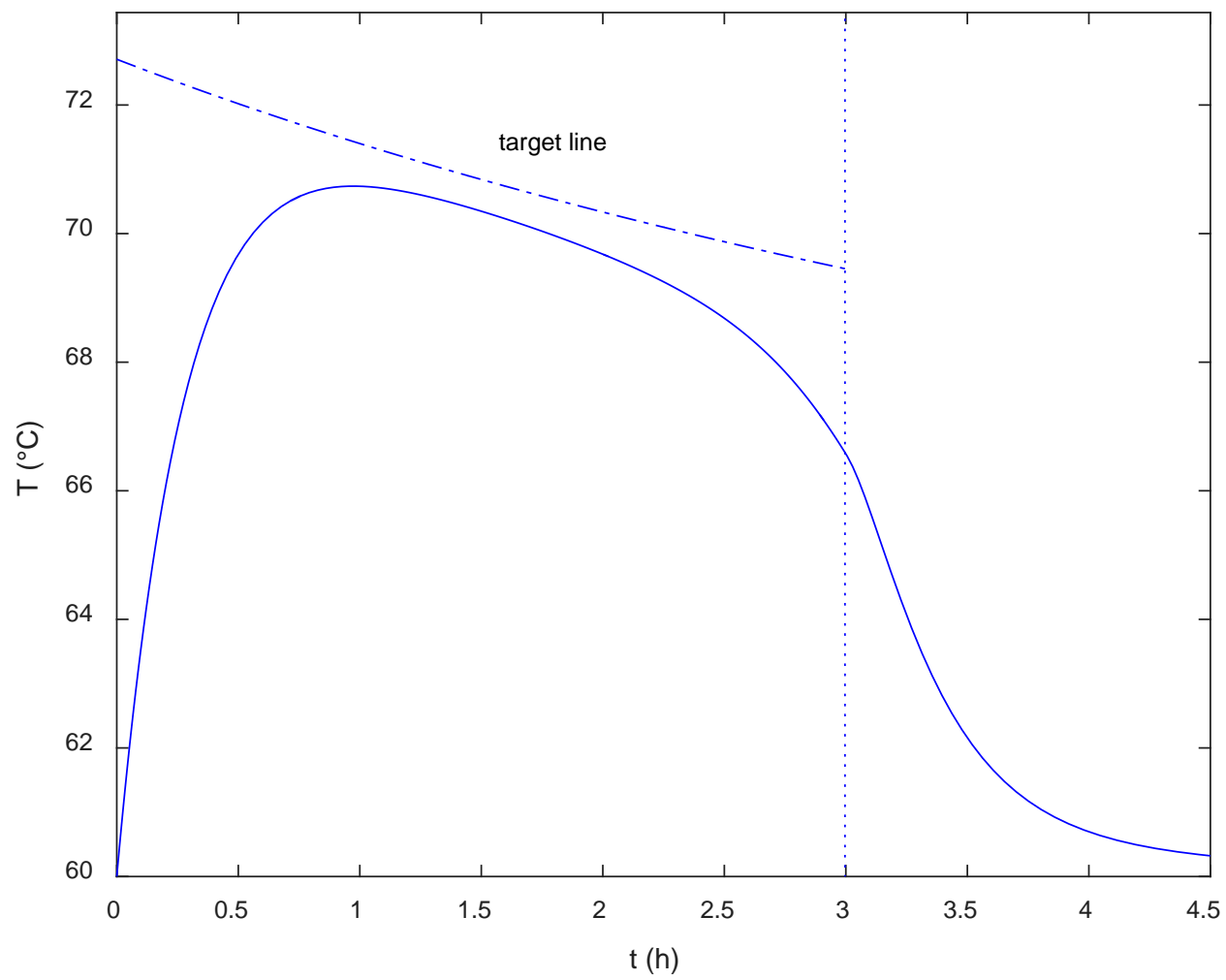

Fig. 1B 


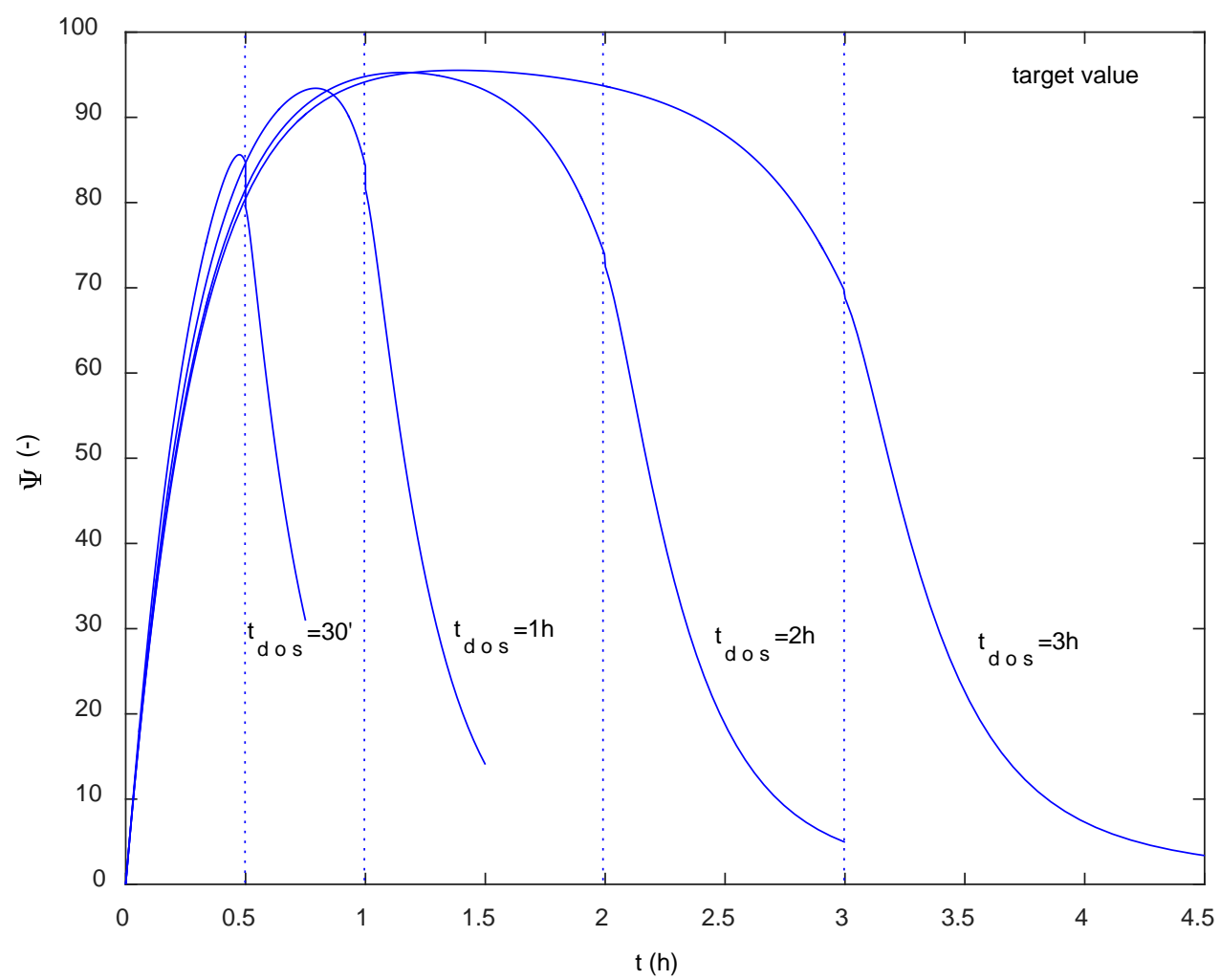

Fig. 2 


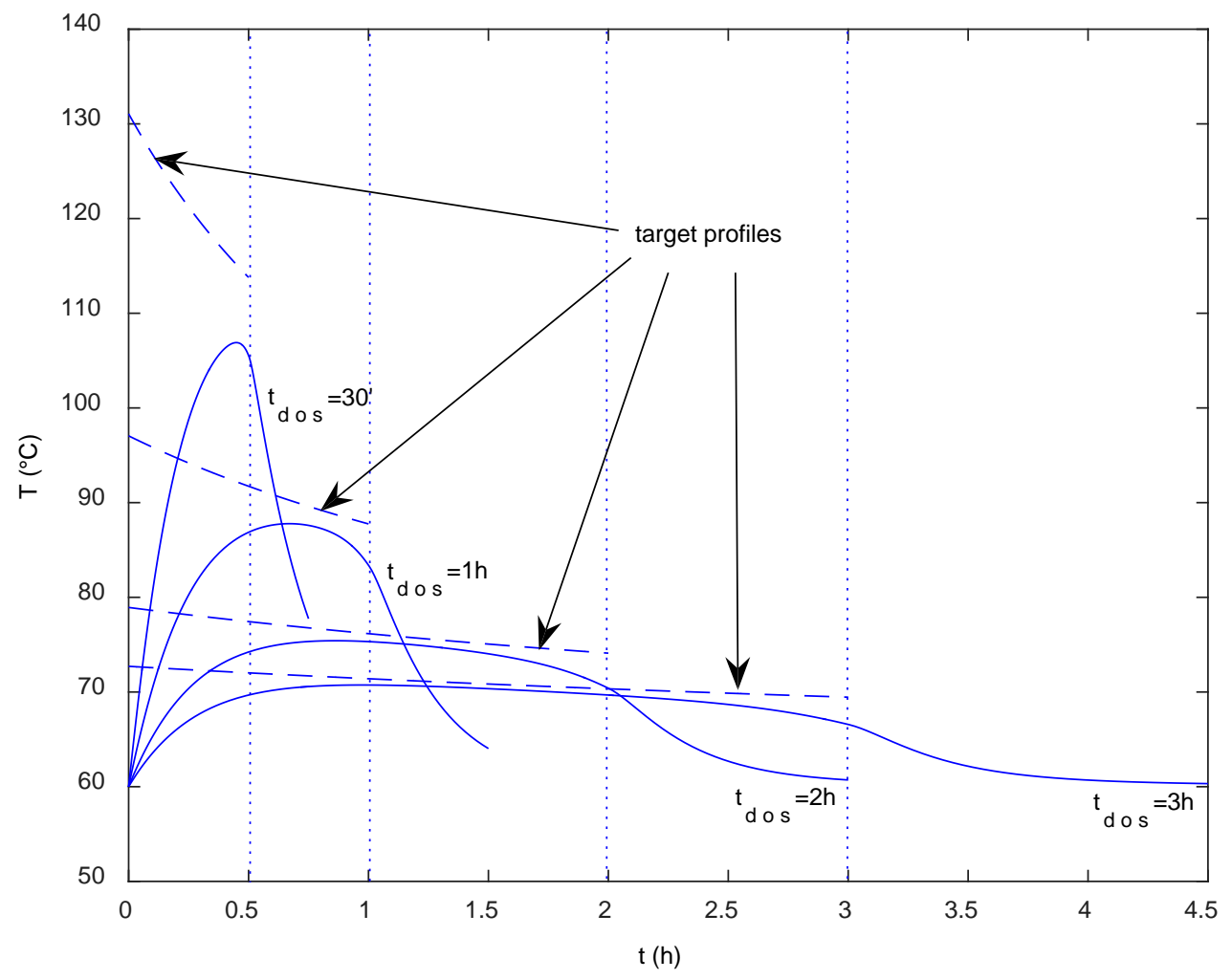

Fig.3 


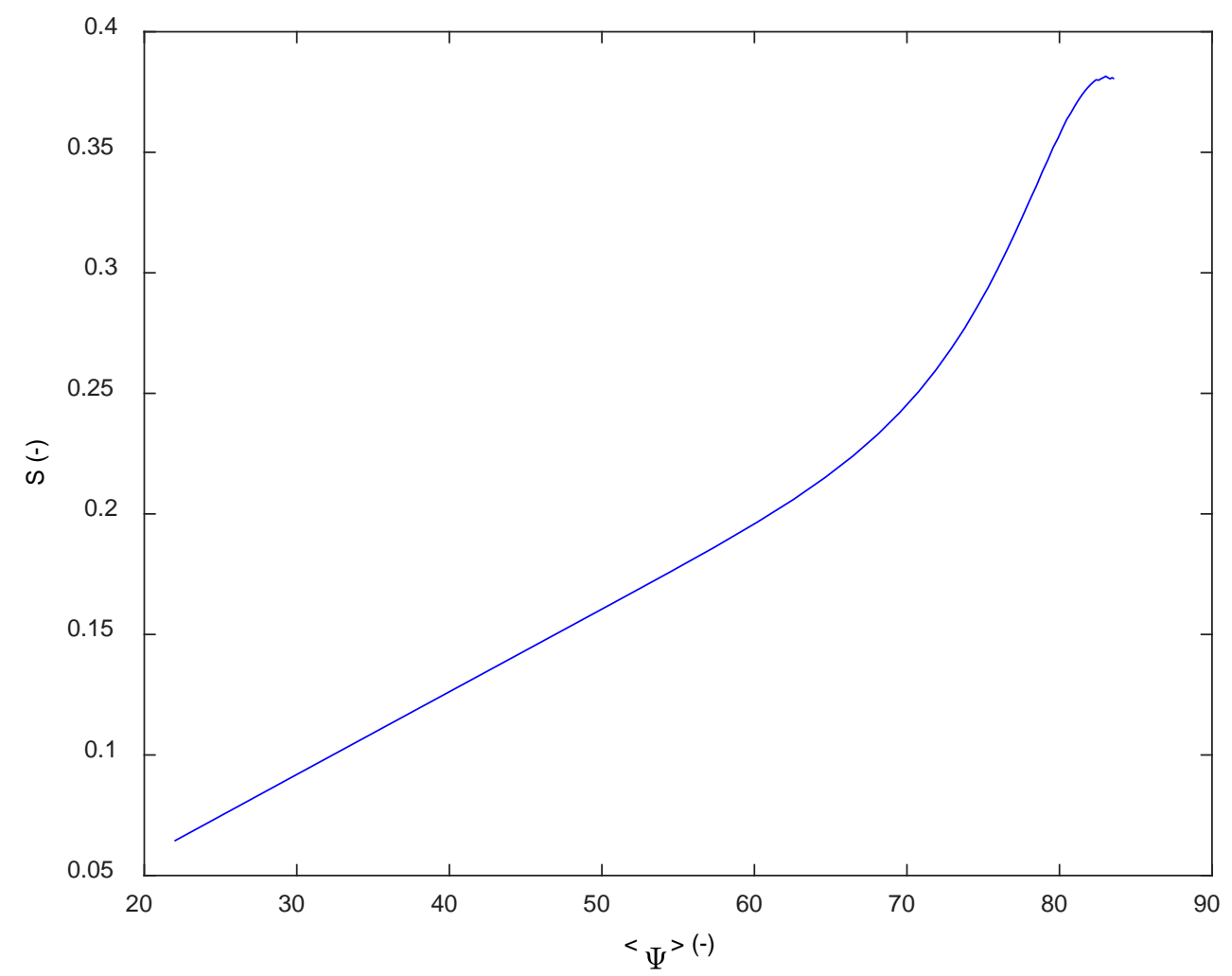

Fig.4 


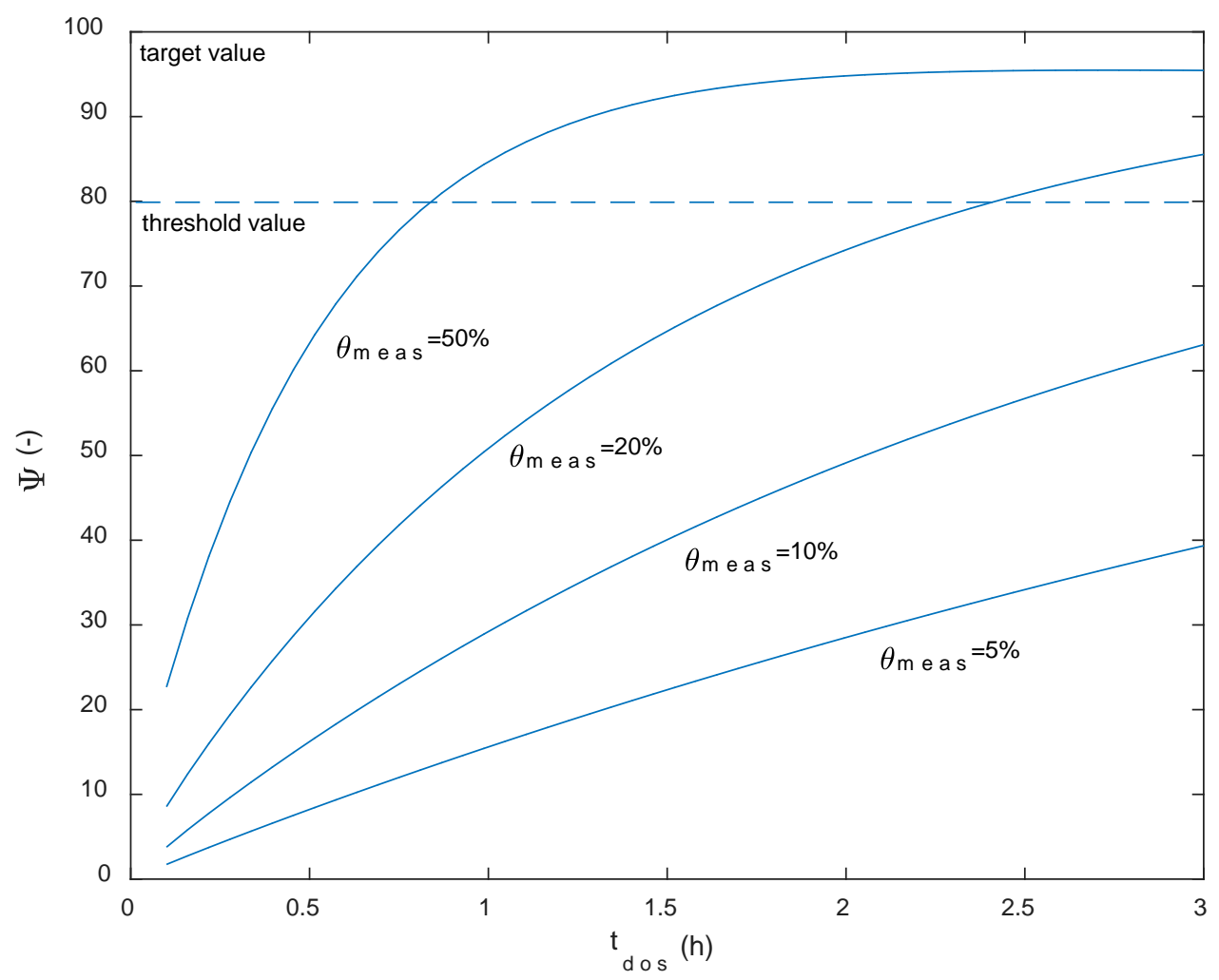

Fig. 5 


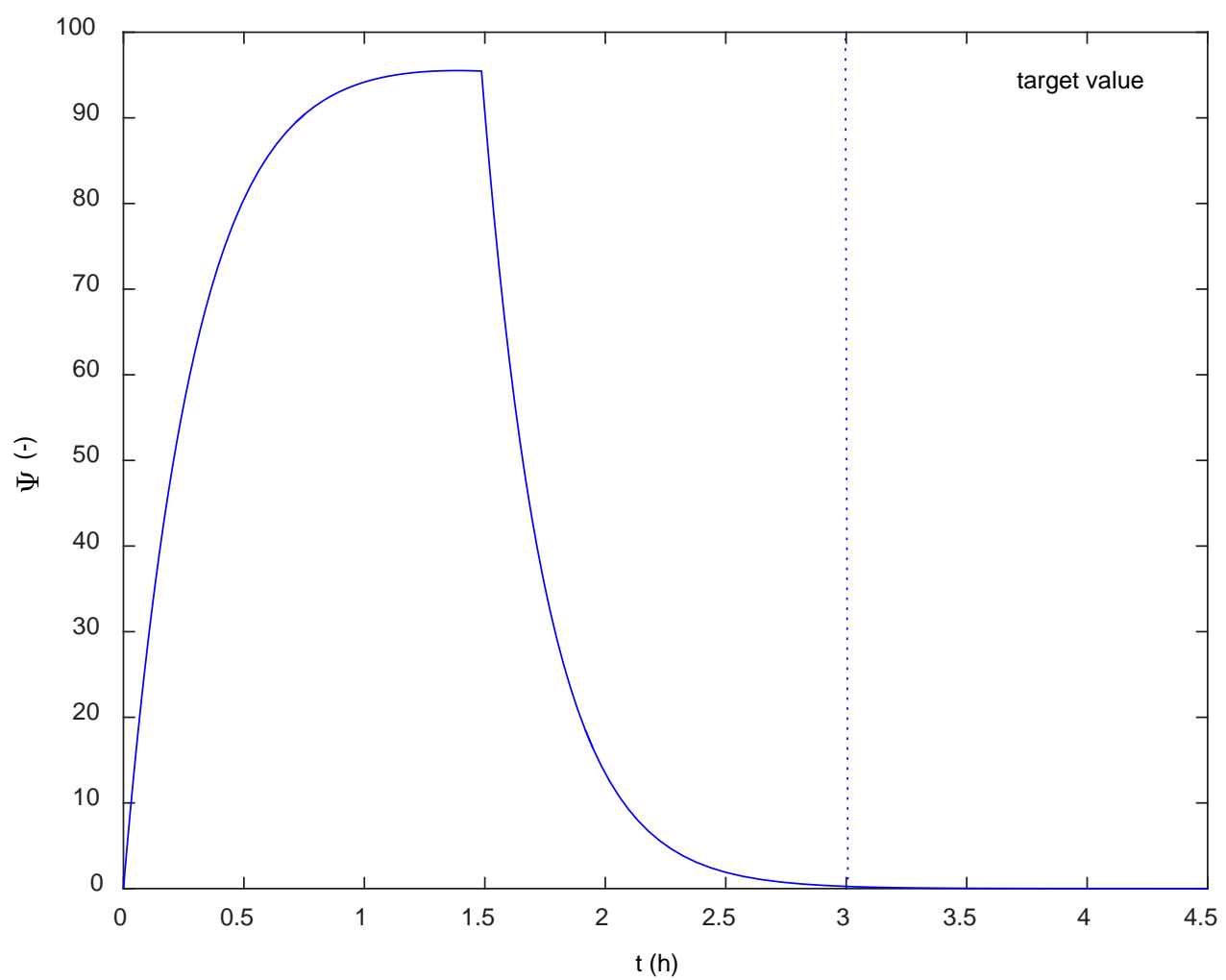

Fig. 6 


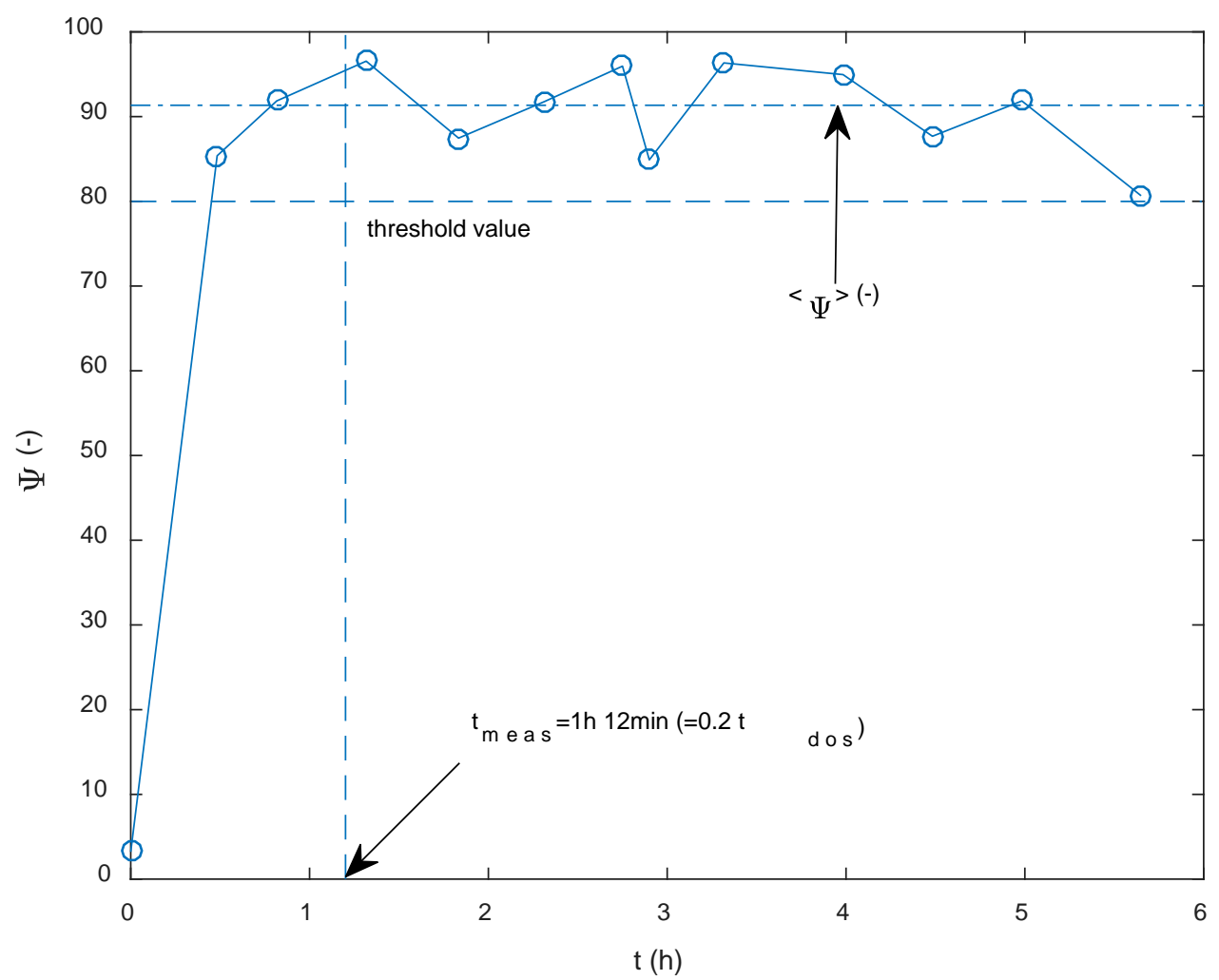

Fig. 7 